\title{
Industrial automation based on cyber-physical systems technologies: Prototype implementations and challenges
}

\author{
Paulo Leitão ${ }^{\mathrm{a}, \mathrm{b}, *}$, Armando Walter Colombo ${ }^{\mathrm{c}, \mathrm{d}}$, Stamatis Karnouskos ${ }^{\mathrm{e}}$ \\ a Polytechnic Institute of Bragança, Quinta Sta Apolónia, Apartado 1134, P-5301-857 Bragança, Portugal \\ ${ }^{\mathrm{b}}$ LIACC-Artificial Intelligence and Computer Science Laboratory, R. Campo Alegre 102, 4169-007 Porto, Portugal \\ ${ }^{\mathrm{C}}$ University of Applied Sciences Emden/Leer, Emden, Germany \\ ${ }^{\mathrm{d}}$ Schneider Electric Automation GmbH, Ratingen, Germany \\ e SAP, Karlsruhe, Germany
}

\section{A R T I C L E I N F O}

\section{Article history:}

Received 23 April 2015

Received in revised form 7 July 2015

Accepted 11 August 2015

Available online 11 September 2015

\section{Keywords:}

Collaborative automation

Cyber-physical systems

Multi-agent systems

Service-oriented architectures

Self-organization

Next generation of industrial systems

\begin{abstract}
A B S T R A C T
Cyber-Physical Systems (CPS) is an emergent approach that focuses on the integration of computational applications with physical devices, being designed as a network of interacting cyber and physical elements. CPS control and monitor real-world physical infrastructures and thus is starting having a high impact in industrial automation. As such design, implementation and operation of CPS and management of the resulting automation infrastructure is of key importance for the industry. In this work, an overview of key aspects of industrial CPS, their technologies and emerging directions, as well as challenges for their implementation is presented. Based on the hands-on experiences gathered from four European innovation projects over the last decade (i.e. SOCRADES, IMC-AESOP, GRACE and ARUM), a key challenges have been identified and a prioritization and timeline are pointed out with the aim to increase Technology Readiness Levels and lead to their usage in industrial automation environments.
\end{abstract}

(c) 2015 Elsevier B.V. All rights reserved.

\section{Introduction}

In the last couple of decades the traditional paradigms adopted for industrial automation are becoming increasingly inadequate to accommodate emerging technology and business needs of manufacturing players. Changing conditions, constraint industrial companies running their business, as they face strong pressure related to the cost, quality and customization of products in highly flexible and responsive production systems [1]. This market and business evolution is generating a need for more flexible and scalable production systems which should be able to handle agile fluctuation with highly product variability at reasonable cost with real-time reactivity. The "collaborative automation" paradigm [58] is a major one supported by the industry, where the aim is the development and implementation of tools and methods to achieve flexible, reconfigurable, scalable, interoperable network-enabled collaboration between decentralized and distributed embedded devices and systems. This trend has been accompanied by a

\footnotetext{
* Corresponding author at: Corresponding author at: Polytechnic Institute of Braganca, Eletrical Engineering, Campus Sta Apolonia, 5301-857 Braganca, Portugal. Tel.: +351 273303083; fax: +351 273313051.

E-mail addresses: pleitao@ipb.pt (P. Leitão), awcolombo@technik-emden.de (A.W. Colombo), stamatis.karnouskos@sap.com (S. Karnouskos).
}

technological evolution characterized by the penetration of computational capabilities, i.e., data and information processing, into the mechatronics, transforming gradually the traditional shop floor into an ecosystem, where networked systems are composed by smart embedded devices and systems, as well as by customers and business partners in business and value processes, interacting with both physical and organizational environment, pursuing well-defined system goals.

Under the label of Cyber-Physical Systems (CPS) and more precisely their applicability in the industrial domain - hence referred to as Industrial Cyber-Physical Systems (ICPS) - key innovation actions have been started in various programs, e.g., the Industrial Internet [2] and German "Industrie 4.0" initiative [3-5]. Communities in several domains are actively working towards designing, implementing and assessing suitable engineering approaches for the realization of the CPS. These approaches are supported by enhancing and developing the necessary technological basis to facilitate the realization of the addressed trends in Industrial Automation, as well as in other emerging application domains, such as smart grids, smart buildings, smart transportation, smart healthcare, and particularly in smart manufacturing [6,7].

In this context, this paper introduces the major features of a set of industrial CPS prototype implementations supporting the realization of the collaborative automation paradigm [8] based 
on the use of Multi-Agent Systems (MAS) and Service-Oriented Architectures (SOA), enriched with insights from social and biological systems, such as swarm intelligence, self-organization and chaos theory. Moreover, some complementary technology enablers, such as wireless sensor networks, augmented reality and cloud computing, are considered to support the operation of the ICPS in ubiquitous environments, where the re-configuration appears naturally like "drag-and-drop" applications and complexity is handled by background services.

The gathered experience in several European research and development (R\&D) projects is used to illustrate some of the achievements in the area and to address new challenges. Particularly, the major results of four selected European Innovation projects are reported i.e.:

- The European Innovation Project SOCRADES - Service-oriented Cross-layer Infrastructure for Distributed Smart Embedded Devices - [9], highlighting the introduction of the serviceoriented architecture paradigm into the industrial automation environment.

- The European Innovation Project GRACE - inteGration of pRocess and quAlity Control using multi-agEnt technology - [10] highlighting the use of agent technology to integrate quality and process control aiming the improvement of the production efficiency and product customization.

- The European Innovation project IMC-AESOP - Architecture for Service-oriented Process, Monitoring and Control - [11], highlighting the application of the service-oriented paradigm to develop and implement the next-generation industrial SCADA (Supervisory Control and Data Acquisition) and DCS (Distributed Control Systems) systems.

- The European innovation project ARUM - Adaptive Production Management - [12], highlighting the integration of agent-based planning and scheduling tools using the service-oriented paradigm to respond faster to unexpected events during the ramp-up production of complex and highly customized products.

This paper is organized as follows. Section 2 describes the basics of the CPS paradigm as a suitable approach to implement the vision for the smart and adaptive factories for the future. Section 3 introduces an high-level view to realize industrial CPS solutions based on MAS principles combined with several technologies, namely SOA and cloud systems, and considering biological inspiration. Section 4 describes four use cases where these concepts were prototype implemented in real industrial scenarios, summarizing the Technology Readiness Levels (TRL) achieved by these industrial prototypes. Section 5 makes a comparative analysis of these use cases and identifies some challenges for approaching the increase of those TRLs. Finally, Section 6 rounds up the paper and presents the conclusions.

\section{The emergence of CPS}

The term "Cyber-Physical Systems" (CPS) coined in 2006 in a high-level working group ${ }^{1}$ composed of selected experts from the USA and European Union, advocates the co-existence of cyber and physical elements with a common goal. Embedded systems have been developed over the past decades, however CPS explicitly pose a focus on the integration of computation with physical processes [13]. Generally, CPS are nowadays designed as a network of interacting cyber and physical elements.

\footnotetext{
${ }^{1}$ NSF Workshop on Cyber-Physical Systems, October 16-17, 2006, Austin, Texas, http://varma.ece.cmu.edu/cps/.
}

In the last years, requirements and overall complexity in the areas of utilization of CPS has increased dramatically. The later is correlated to the pursuit for flexibility, customization, interaction and provision of new functionalities in industrial settings. Currently, there is a technology push into complexity, with everything getting smart, e.g., phones, houses, cars, aircrafts, factories, cities etc. As an example, the functionalities and consequently complexity associated can be seen by a system comparison e.g. of the early past century plane controls in Charles Lindbergh's "Spirit of St. Louis" and a modern Airbus A380 aircraft. Although both have the common goal of flying, this could be realized by monitoring a couple of sensors in the "Spirit of St. Louis", which nowadays translates to thousands of sensors in A380, which is impossible to assess for humans. However, with the automation and creation of high-level key performance indicators from the sensor data, this can still stay manageable at high level, although not all interworking are directly seen nor understood by its operators. Although complexity may have its advantages, hiding it from the end-users and managing it, results to grand challenges. As an example, in our cars, the complexity is hidden from the driver, as she/he just needs to handle a limited number of controls to operate the system, without being exposed to its complex networks of sensors and actuators distributed all through the mechanic infrastructure.

Particularly in manufacturing automation, markets are imposing strong changing conditions, where the customization of products requires the use of flexible automation infrastructures. Moreover, the application of flexible automation cannot completely guarantee respecting the time to market requirements, compared with the usual short time on the market of the manufactured products. This situation lies to the necessity of developing and implementing, in a complementary manner to the addressed flexibility, fast and manageable reconfigurability of the automation systems. This means, the reconfigurability of mechatronic (physical part of the automated objects) and of the automation software (cyber part of the automated objects).

As depicted in Fig. 1 (adjusted from Ref. [14]), there are several areas that share common ground, e.g., software agents, Internet of Things, CPS, cooperating objects etc. These have co-evolved over the last decades, and although some of these are used interchangeably (in places), there are differences among them. In our view, what differentiates them is the varying mix of the degree of physical and feature elements that creates the right recipe for a specific area. For instance, Cooperating Objects [14] focus mostly on the cooperation aspects while considering the rest of the available features only as enabling factors to achieve cooperation. Other approaches, e.g., Internet of Things, focus mostly on the interaction and integration part while cooperation is optional. Similarly, CPS may pose a different mix of the key features and depend on their utilization domain. CPS consider the computational decisional components that use the shared knowledge and information from physical processes to provide intelligence, responsiveness and adaptation. In conclusion, the differentiating factor among all areas, is not the distinct characteristics but which of them they employ (depending on the scenario) and at which degree.

CPS in industrial infrastructures deal also with the combination of mechatronics, communication and information technologies to control distributed physical processes and systems, designed as a network of interacting software and hardware devices and systems, many of them with a higher level of decision-making capabilities in both aspects: "autonomic" with self-decision processes [15] and "collaborative" [14] with negotiation-based decision processes. CPS can be considered as smart systems that use cyber technologies embedded in and interacting with physical components, featuring a tight combination of computational and physical elements, integrating computation, communication and control over an information system (integration of computation 


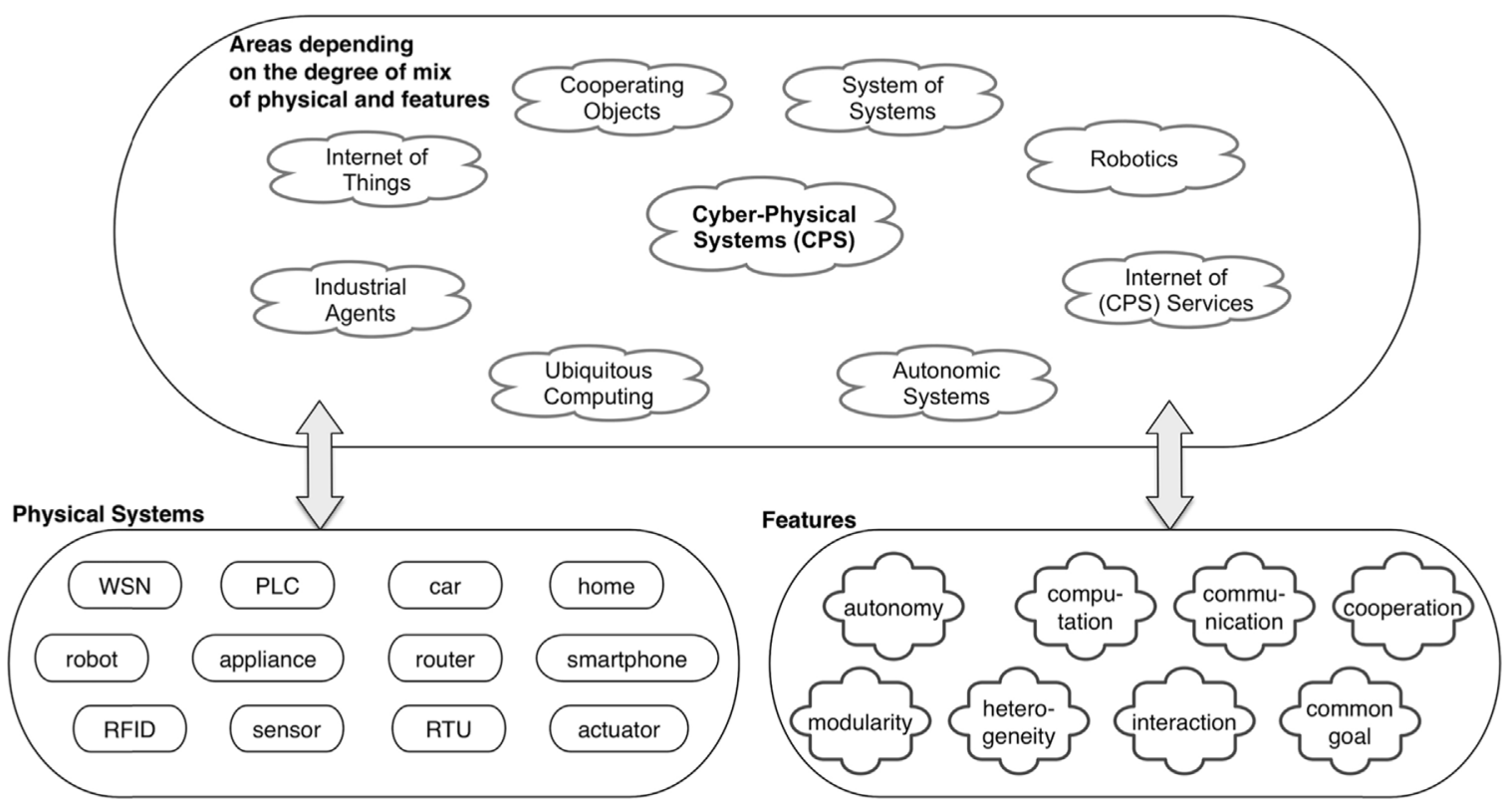

Fig. 1. The mix of physical systems and features as a basis for CPS.

and physical processes). The concept of CPS extends the concept of embedded systems: in embedded systems the focus is on computational elements hosted in stand-alone devices, while CPS is designed as a network of interacting computational and physical devices.

\section{Engineering CPS}

The engineering aspects of CPS are highly challenging, as CPS aim to realize the evolution from traditional, rigid approaches into decentralized structures, implementing the "Industrie 4.0" and Industrial Internet visions. Therefore, the deployment of CPS in the industrial environment is a critical issue, requiring proper methodologies that will enable the high readiness of these systems for the industrial usage. Fig. 2 illustrates a high level view for the deployment of CPS, centered on using distributed intelligence (e.g. realized by multi-agent systems), allowing distributing a complex problem into a network of modular, intelligent, adaptive and pluggable components with the intelligent global behavior emerging from the interaction among the individual components. The use of holonic principles simplifies the system's design by using several important characteristics, such as recursivity and hierarchies formed by intermediate stable forms.

Another dimension is also introduced by combining multi-agent systems (MAS) with complementary technologies to overcome some limitations of MAS solutions, namely the interoperability in

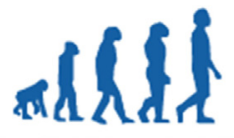

Adaptation and evolution (self-* properties, chaos theory, ...)

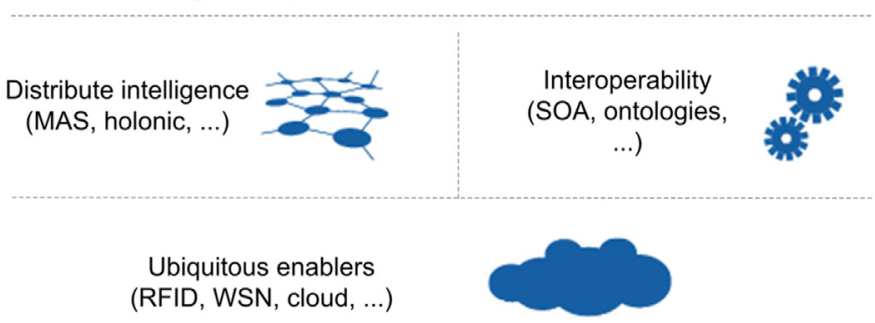

Fig. 2. High-level view for the deployment of Cyber-Physical Systems. vertical and horizontal integration and the integration with low level control. For the first case, MAS can be integrated with SOA forming a service-oriented multi-agent system, which not only share services as form of communication, but instead constitutes a network of distributed agents that integrate SOA principles. In such systems, the front-end layer comprises services encapsulating the functionalities provided by the agents, which by their turn provide control, intelligence and autonomy. For the second case, since MAS usually misses real-time constraints, additional efforts e.g. in the IEC 61131 standard can be integrated to preserve the low-level control to ensure responsiveness, while agents provide intelligence and adaptation at higher control level.

This high control layer can be enriched by embedding social and biological inspired techniques, which provide plenty of simple but powerful mechanisms to handle complex environments, allowing to develop truly self-adaptive and evolvable complex systems. For this purpose, emergence, swarm intelligence, chaos theory and particularly self-organization concepts will be integrated with the CPS world. An important issue is also the use of technology enablers to support the operation of CPS in ubiquitous environments, where re-configuration appears as a natural issue, and complexity is handled by background services. Here the use of augmented reality, cloud computing and fog computing are necessarily important to be considered and the use of Big Data infrastructures and techniques will play a significant role for the data analytics in the shop floor and/or collaborative supply chains.

\subsection{Key CPS technologies: Agents, SOA, cloud}

CPS are centered on the use of several technologies, namely MAS, SOA and cloud systems.

\subsubsection{Agents}

The first step is to use Multi-Agent Systems (MAS) [16-18] to achieve distributed intelligence and adaptation. An agent can be defined as "an autonomous component, that represents physical or logical objects in the system, capable to act in order to achieve its goals, and being able to interact with other agents, when it doesn't possess knowledge and skills to reach alone its objectives" [18]. Since rare applications consider agents in an isolated manner, these systems form multi-agent systems, concept inherited from 

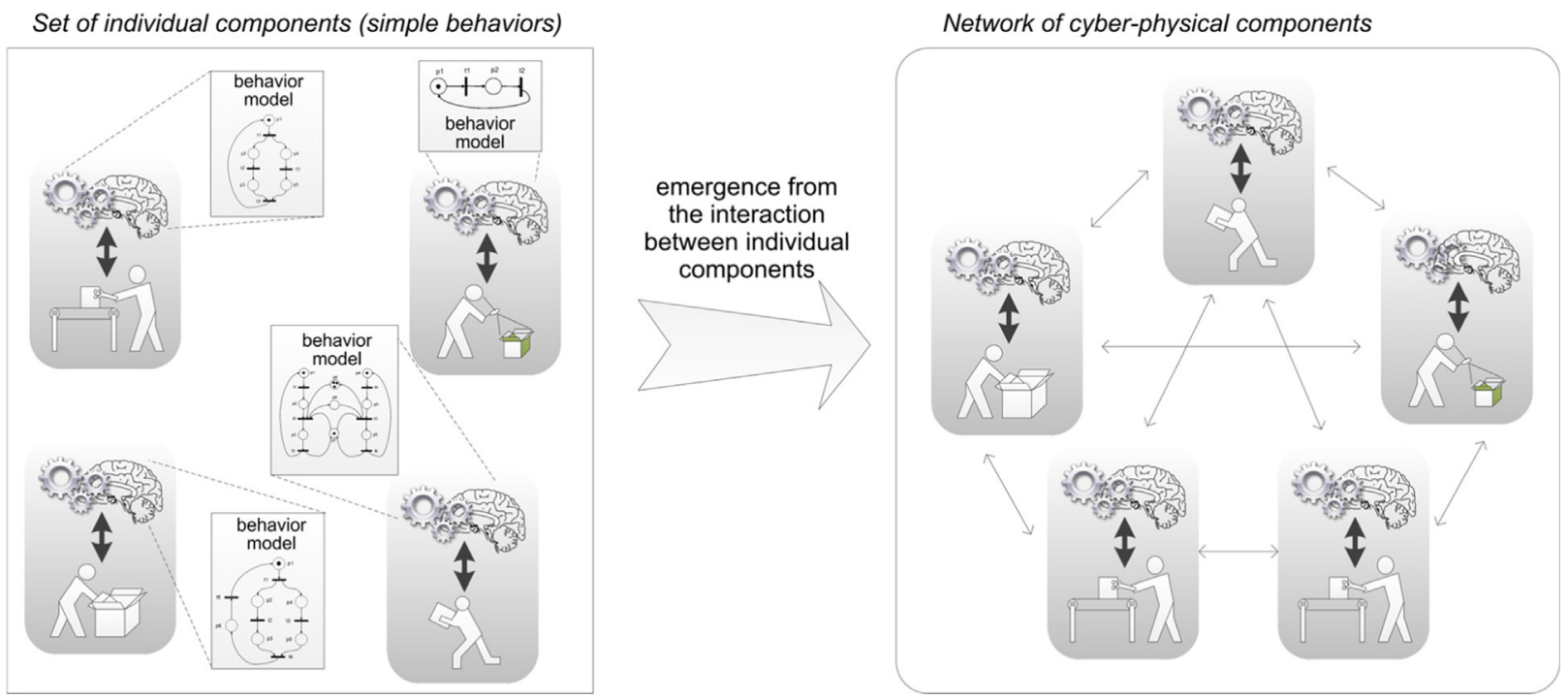

Fig. 3. Multi-agent system for manufacturing control.

distributed artificial intelligence field, which can be defined as "a society of agents that represent the objects of a system, capable of interacting to achieve their individual goals when they have not enough knowledge and/or skills to achieve individually their objectives" [18].

In such network of distributed, modular, intelligent and pluggable agents, the intelligent and global behavior emerges from the interaction among agents, with each individual agent contributing with its knowledge and skills, as illustrated in Fig. 3. MAS offer an alternative way to design complex systems based on the decentralization of functions, providing modularity, flexibility, robustness, adaptability and re-configurability. Especially in industrial settings, the emergence of industrial agents [19] focuses on the introduction of intelligence that can be performed in automation devices (e.g. sensors, actuators, robots and machines), systems and infrastructures, effectively enabling the creation and interaction of cyber-physical components/systems.

In such distributed and heterogeneous systems, the knowledge sharing can be a problem, since each agent has its own knowledge structure, lacks of understanding of exchanged knowledge which affects the inter-operability and the reuse and share the knowledge. The solution is to use ontologies [20] to represent the structure of the shared knowledge, allowing distributed agents to understand themselves during the cooperation processes.
The design of these distributed systems can use the holonic principles, which considers complex systems build upon hierarchical systems formed by intermediate stable forms that do not exist as auto-sufficient and non-interactive elements [21]. A crucial piece is the concept of holon that simultaneously represents a part and a whole, i.e. is simultaneously a self-contained whole to its subordinated parts and a dependent part when seen from higher levels [22-24]. Taking advantage of the holon nature, and mainly the Janus effect, the use of holonics simplifies the system's design by using the recursivity property. As example, the holonic principles can be used to design a network of schedulers: a scheduler for a station comprises a set of agents representing individual workers; this station scheduler can also be part of a bigger scheduler representing a workshop, and successively to represent a factory comprising several workshops.

Since CPS require the integration of computational systems with physical devices, it is mandatory to interconnect agents with physical hardware devices. However, MAS usually miss real-time constraints. A suitable approach is preserve the low-level control based on the state-of-the-art PLC (Programmable Logic Controller) programs using IEC 61131-3 and IEC 61499 standards to ensure responsiveness, and develop MAS on a higher-level control to provide intelligence and adaptation, as illustrated in Fig. 4, which is adapted from Marik and McFarlane [25].

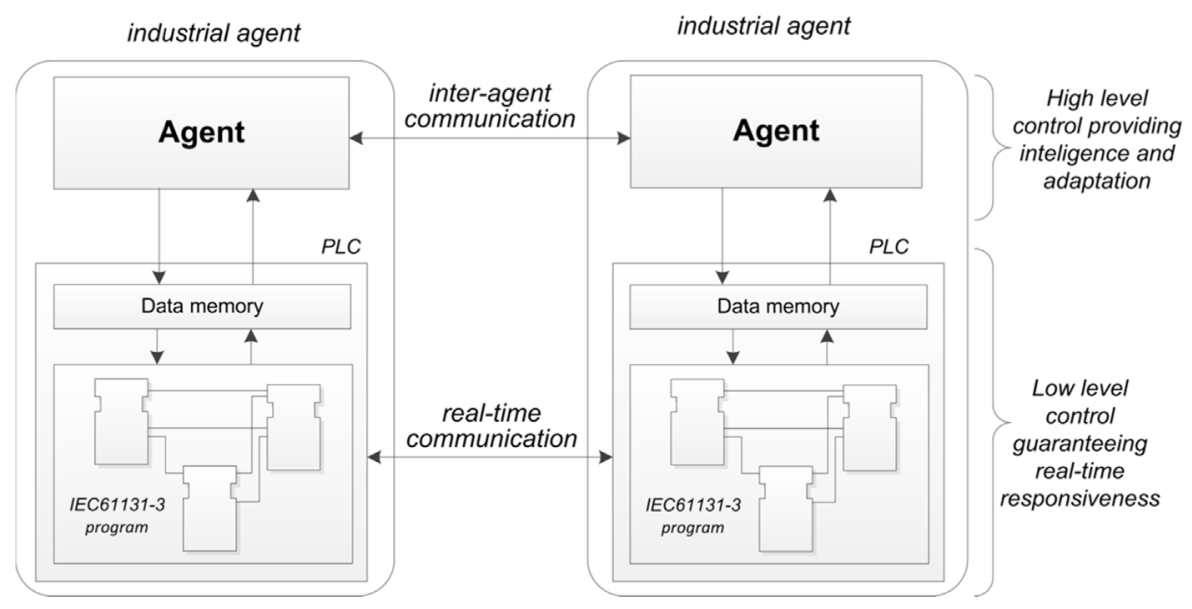

Fig. 4. Combining agents with IEC 61131-3. 
This integration solution allows the deployment of intelligent cooperative objects (agents) in physical controllers and recalls the holonic concept (note that an important characteristic is that holons represent computational and physical parts [26], which matches perfectly with the cyber-physical components structure). The challenge is related to how to standardize the structure of messages between the agent and the PLC programs.

\subsubsection{Services as enabler of CPS}

The second step considers the need to combine MAS with complementary technologies to overcome some of its limitations, namely in terms of interoperability. The integration of agents and SOA using agents, to embed the intelligent logic control that is exposed as services to the other agents, enables the adoption of a unifying technology for all levels of the enterprise [27,28]. The pertinent question that arises from this discussion is how to combine MAS and SOA and apply them effectively in industrial scenarios [27]. The first option is to use gateways to translate the semantics from the agents to the services worlds. Basically, these gateways translate the:

- Service registration: the Directory Facilitator defined in FIPA (Foundation for Intelligent Physical Agents) versus UDDI (Universal Description, Discovery and Integration) defined in OASIS.

- Service description: the agent skills versus WSDL (Web Service Definition Language).

- Message: the ACL (Agent Communication Language) versus SOAP (Simple Object Access Protocol).

Examples of such gateways are the Web Services Integration Gateway (WSIG) and AgentWeb Gateway, the first one being a JADE [29] add-on. However, a more suitable approach is to consider Service-oriented Multi-Agent Systems (SOMAS) [30], which not only share services as form of communication but instead is a network of distributed agents that integrate the SOA principles. The usage of software agents in industry has a long history, and with the emergence of services, cloud and CPS, new capabilities and challenges arise [19]. However, to what extend these will prevail, will highly depend on the fulfillment of the requirements stemming from the key factors that enable industrial agent acceptance [31]. Note that several surveys and studies have analyzed the possible barriers that constraints a wider industrial adoption of the agent technology in industrial environments [18,31-33].

In this way, a front-end layer is established with services encapsulating the functionalities provided by the agents, which provide control, intelligence and autonomy, as illustrated in Fig. 5. This approach provides modularity in a similar manner to the Lego ${ }^{\mathrm{TM}}$ concept, allowing to build complex distributed systems as building blocks.

In such service-oriented systems, some services can be composed with other atomic services exposing aggregated functionalities and offered to other agents. For this purpose, the introduction of components exposing orchestration and choreography capabilities as services into the system is crucial to be executed.

A general engineering methodology to developing and implementing a SOA-based automation platform for CPS will start with the specification of elementary services dedicated to the device and system hardware-specifications, will continue with the specification and implementation of "application services" addressing atomic automation functionalities and will be completed with the specification and implementation of methods and tools enabling the composition, orchestration and sometimes choreography of those atomic services. One of the major results of this last stage of the methodology is the effect that devices and systems that were developed for very explicit and dedicated automation functionalities, are now able to offer complementary functionalities only due to the fact that they are having an embedded orchestration/choreography engine and it adds these new functionalities as services resulted from the service-composition [9].

\subsubsection{Cloud-based services as enabler for CPS}

The last years we have witnessed the rise of cloud computing [34] as a new way to develop and deploy sophisticated applications

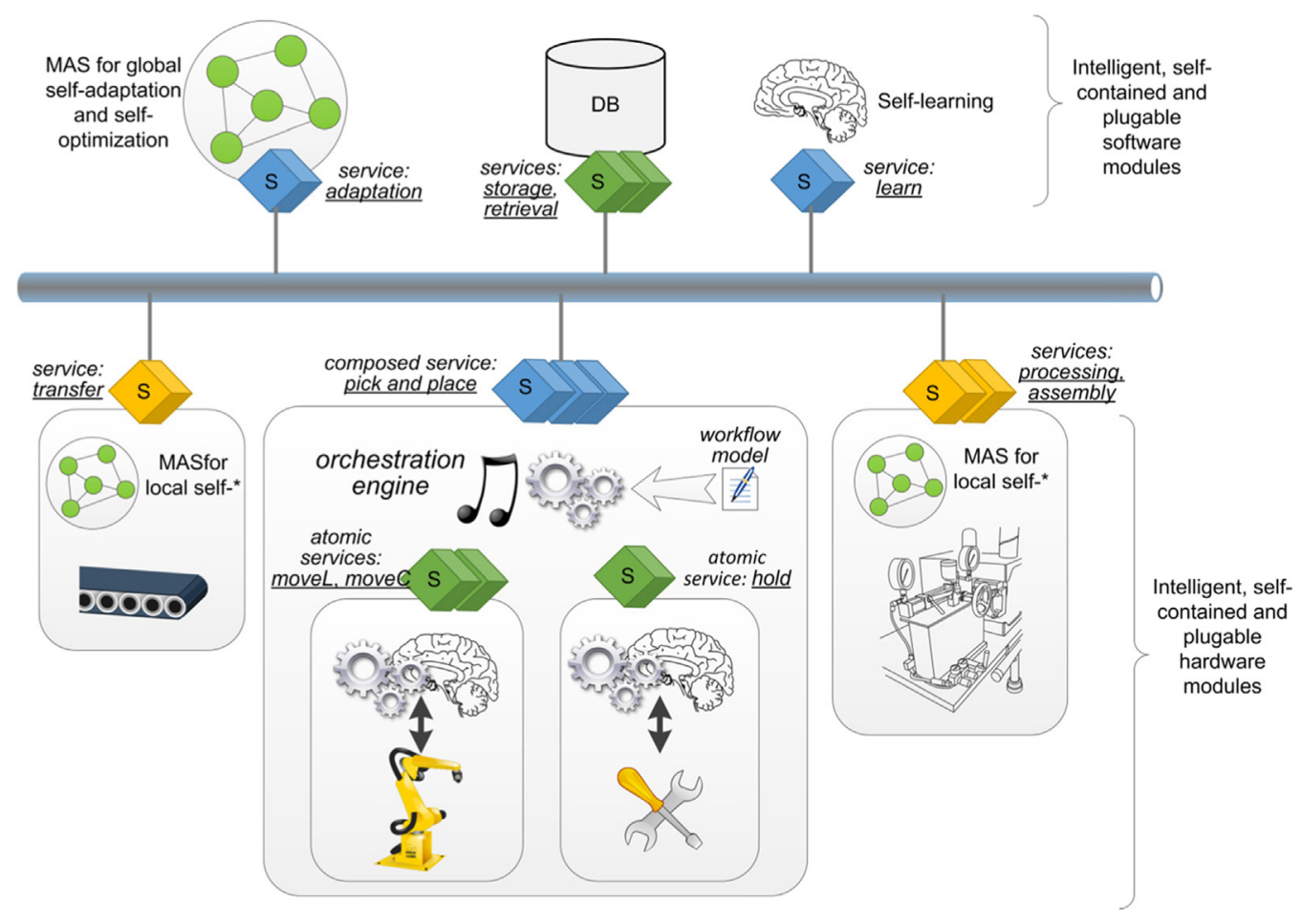

Fig. 5. Service-oriented multi-agent system. 
at scale. In addition, the elasticity of resources provided by the cloud, have acted as an enabler for industrial scenarios, not only on the infrastructure integration level, but also on massive data storage and high-performance analytics on it. In the era of cyberphysical system dominated industrial infrastructures, a huge volume of data is collected in real time by a vast number of networked sensors that need to be analyzed in real time. Big Data [35] and the real-time analytics applied on Big Data in cloud systems, enables the implementation of these techniques to extract new insights from the data. Several industrial applications already utilize cloud-based architectures and services [11,19].

The trend towards virtualization of resources and key aspects of real-world processes addresses many enterprise needs for scalability, more efficient use of resources, and lower Total Cost of Ownership (TCO), just to name a few. Cloud Computing has been widely adopted by the industry as it enables grasping the benefits of virtualization, SOA and utility computing. CPS services are accessed over the Internet but still offer to the application the feeling as if they were installed locally. The vast computation and storage resources available in the cloud that can scale out or up to the needs of the specific application, provide a motivating factor for the utilization of cloud computing in industrial scenarios. As such, agents (and agent-based CPS) can now utilize the cloud infrastructure for computationally intensive tasks and delegate such actions (e.g. analytics to real-time data flows) that could enable them to take better decisions. In addition, using the cloud as a communication medium via which interactions are made, has the potential to streamline communication among previously siloed systems that would require significant effort to communicate directly.

Key concerns though with cloud based infrastructures include according to Badger et al. [34] security, privacy, client (browser usually) risks, high dependence on network, side-effects due to multi-tenancy, potential portability issues among cloud layers etc. Although these exist and work is carried out to tackle the issues, this does not mean that they are prohibitive for industrial environments. The tradeoffs however need to be investigated per use-case and stakeholders involved. One thing is clear however, the cloud infrastructure cannot yet adequately support real-time operations and interactions at large. The latter is mandatory for many industrial applications (e.g. robots in a factory), however today it can be approached only with dedicated communication links in private clouds, which however are costly.

The penetration of the SOA, MAS and Cloud Computing paradigms into the automation environment cannot be done without considering the legacy systems that are currently installed, mainly following the ISÁ95 enterprise reference architecture specifications (www.isa95.org). This includes a "migration process" from a traditionally hierarchical management and control infrastructure to a heterarchical and distributed one. This migration process consists on step-by-step migration approach for dedicated components of the hierarchical infrastructure, whose functionalities will firstly be transformed in "service-providers" and/or "serviceconsumers", secondly the services will be embedded into legacy or new hardware or exposed into a "service cloud" represented physically by e.g., a "service bus". Such migration aspects when applied to SCADA/DCS components and functions, are discussed more in detail by Colombo et al. (ch.5 and ch7) [11].

The use of ubiquitous environments and integration of CPS and alike approaches allows for intelligent approaches to flourish. For instance, the use of the RFiD technology, and especially its future evolution as nanotechnology in products or resources, will support the realization of intelligent products [36] and large-scale tracking in automation systems. Other emerging concepts such as Fog computing [37], are similar to cloud systems, providing data, computation, storage and application services near to the point of action i.e. with proximity to end-users and devices. The latter are geographically distributed over heterogeneous platforms, being sensitive to real time interaction, allowing to control critical resources like energy, traffic and health-care. Fog computing complements the use of cloud to overcome the different real time scopes in a CPS environment.

Finally, in such industrial infrastructures, humans are seen as important stakeholders and CPS being human-centered technology plays a special role [38]. The interaction with humans can be achieved via the use of mobile devices, such as smartphones, tablets or, more recently, the wearable devices, that can act as the interface for a bidirectional communication. The augmented reality technology can be combined with agents to support operators during the installation, operation and maintenance of automation systems. This enhances the productivity and efficiency of operators by providing information related to historic and current status of the device/system and providing additional information, e.g., documentation, web pages or videos. Google Glass is an example of such augmented reality technology that can be used in industrial shop floor environments.

\subsection{Building sophisticated systems}

An important aspect in engineering the next generation of CPS, might be to consider social and biological inspiration to develop truly self-adaptation and evolution of complex systems. Biology offers a plenty of simple but powerful mechanisms to handle complex environments [39] and the idea is to translate these mechanisms to solve complex manufacturing problems and not simple copy them (an usual mistake when considering the biological inspiration).

An interesting mechanism is the swarm of intelligence where the global behavior emerges from the contribution of simple individual entities. A swarm is formed by a community of homogeneous entities regulated by simple rules and exhibiting mainly reactive behavior, without the presence of central authority [40]. In such swarms, it is usually used coordination of activities using simple feedback mechanisms instead of complex decisionmaking procedures. Examples can be found in ant and birds colonies, and also in Wikipedia which is somehow a kind of swarm intelligence.

In such environments, the resulting behaviors and patterns are more complex than the individuals from which they emerge, i.e. more than the simple sum of individual behaviors [41]. The complexity also comes from the sensitivity to initial conditions, known as butterfly effect, and the non-linear interactions involving amplification and cooperation. In this way, the emergent behavior is difficult to predict being important to ensure that the unexpected and undesired properties or behaviors will not emerge.

Self-organization [42] is an evolution mechanism where, new and complex structures take place primarily through the system itself, normally triggered by internal variation processes. In such systems, an important issue to be considered is to control nervousness avoiding to have a system working in a chaotic state. Several examples of self-organization can be found, e.g., birds, fishes, even in some chemical reactions and also in some traffic jams. In manufacturing, the self-organization concept can be translated for the development of self-organized CPS that allow to achieve truly reconfigurable systems better addressing the current industrial requirements, as illustrated in Fig. 6. Several works already experimented the use of self-organization in MAS, such as P2000+ [43], ADACOR [59] and PROSA combined with stigmergy concepts [60].

However, a significant work should be performed to take the entire potentiality from this concept, as it is being pointed out by the $\mathrm{ADACOR}^{2}$ architecture [44] that considers a behavioral and 

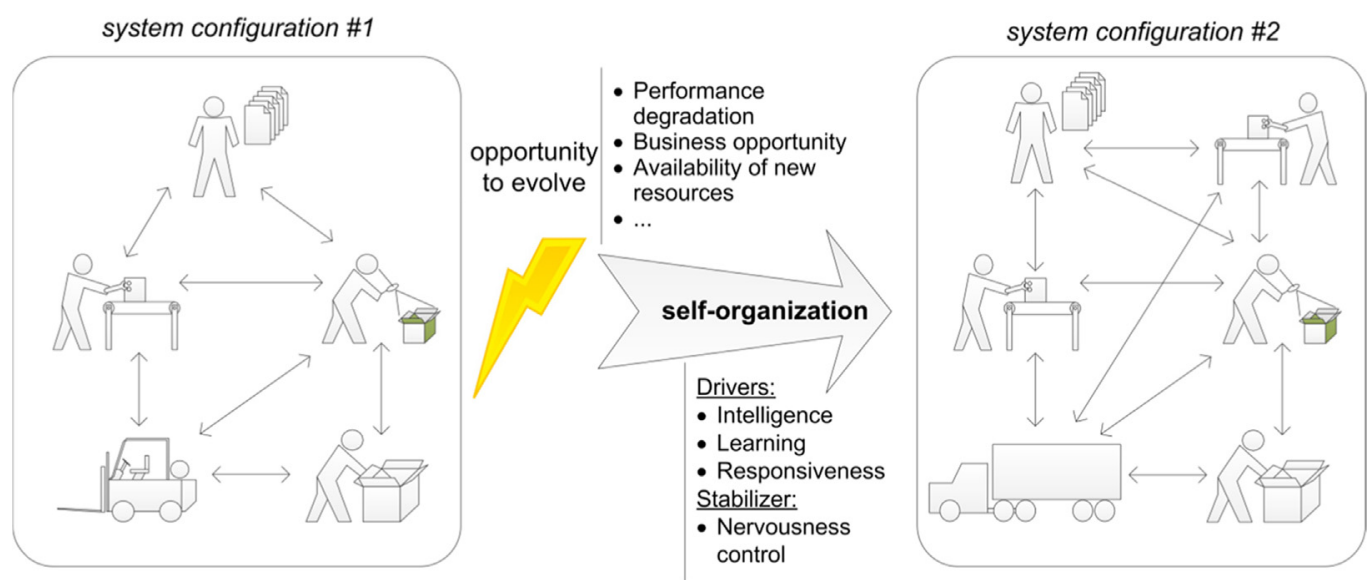

Fig. 6. Self-organization in Cyber-Physical Systems.

structural self-organization approach. In this approach, the behavioral self-organization is related with the smooth evolution of the components and system (aligned with the Darwin's theory of evolution of the species) and the structural self-organization is related to the drastic evolution episodes (aligned with the punctuated equilibrium theory). In such dynamic and selforganized systems, the system nervousness should be controlled aiming to push the system into its limits but remaining in a stable state.

\section{Industrial use-cases}

In the last decade, several research and prototyping efforts have been developed in the domain of industrial CPS in Europe, US and Asia. In this work we will focus on four such industrial projects carried out in Europe, as they had significant industry participation and are seen as lighthouse projects reflecting a wider community thinking and transition. The four case studies selected are the European Commission co-funded projects: FP6 SOCRADES (SOA in Manufacturing Automation), FP7 GRACE (MAS in Manufacturing Automation), FP7 IMC-AESOP (SOA in SCADA and for Process Automation) and FP7 ARUM (MAS combined with SOA for Production Management/MES). Although some information are shortly depicted here, all of these projects have published extensively their results in conferences and journals, hence readers can further deep-dive to their achievements.

The maturity and technology readiness of the industrial prototypes developed in these $R \& D$ projects will be analyzed according to the levels defined by the [45]. TRL are based on a scale from 1 to 9, where TRL 1 is related to basic principles observed, TRL 2 to technology concept formulated, TRL 3 to the experimental proof of concept, TRL 4 to technology validated in lab, TRL 5 to technology validated in relevant environment - industrially relevant environment in the case of key enabling technologies, TRL 6 to technology demonstrated in relevant environment industrially relevant environment in the case of key enabling technologies, TRL 7 to the system prototype demonstration in operational environment, TRL 8 to the system complete and qualified, and TRL 9 to actual system proven in operational environment-competitive manufacturing in the case of key enabling technologies.

\subsection{SOCRADES}

The SOCRADES technical approach adopted the "collaborative automation" paradigm $[8,9,46,58]$ and it is devoted to create a service-oriented ecosystem, where networked systems are composed by smart embedded devices interacting with both physical and organizational environment, pursuing well-defined automation goals. The approach take the granularity of intelligence to the device level of an automation system allowing intelligent system behavior to be obtained by composing, orchestrating configurations of devices that introduce incremental fractions of the required intelligence as "web services". From a functional perspective, the focus is on managing the vastly increased number of intelligent devices and mastering the associated complexity of the physical objects having their digitalized cyber-shadow in the information-communication infrastructure. From a run-time infrastructure viewpoint, the automation system engineer is confronted with a new breed of very flexible real-time embedded objects i.e. automation devices and systems (wired/wireless) that are fault-tolerant, reconfigurable, safe and secure. In this way, for example, auto-configuration management is a new challenge that is addressed through basic plug-and-play and plug-and-run mechanisms facilitated by the physical connectivity and cyberinteroperability.

As illustrated in Fig. 7 [11], SOCRADES components are a kind of CPS whose functionalities are encapsulated as web services and offered to other components through a service bus that supports forming a network of smart components/objects. The shop floor (physical components (hardware/software) is having its cyber-shadow represented in a service-cloud. In this manner, smart embedded automation devices comprise the intelligence and logic control for the mechatronic part and are a source of automation functionalities that can be accessed from any node of the network and any other member/component of the cloud. From technological and infrastructural viewpoints, the use of the SOA paradigm implemented through Web services technologies enables the adoption of a unifying technology for all levels of an industrial enterprise, from sensors and actuators located on the shop floor to enterprise business process. Among others, this means that low cost devices (i.e., in the range of a few Euro) can communicate and exchange data and information (represented as "services") directly to higher-level systems like SCADA, MES and ERP. Within SOCRADES a full-fledged architecture for integration of CPS to enterprise systems and services has been designed, implemented and assessed, as analyzed by Karnouskos et al. [47].

The building blocks of the collaborative automation system [8], which are built upon SOA, will then present their functionalities and production operations as Web services inside the building block network (service cloud) and form the desired production process by collaborating using the communication and information-exchange methods provided by the web technology. 

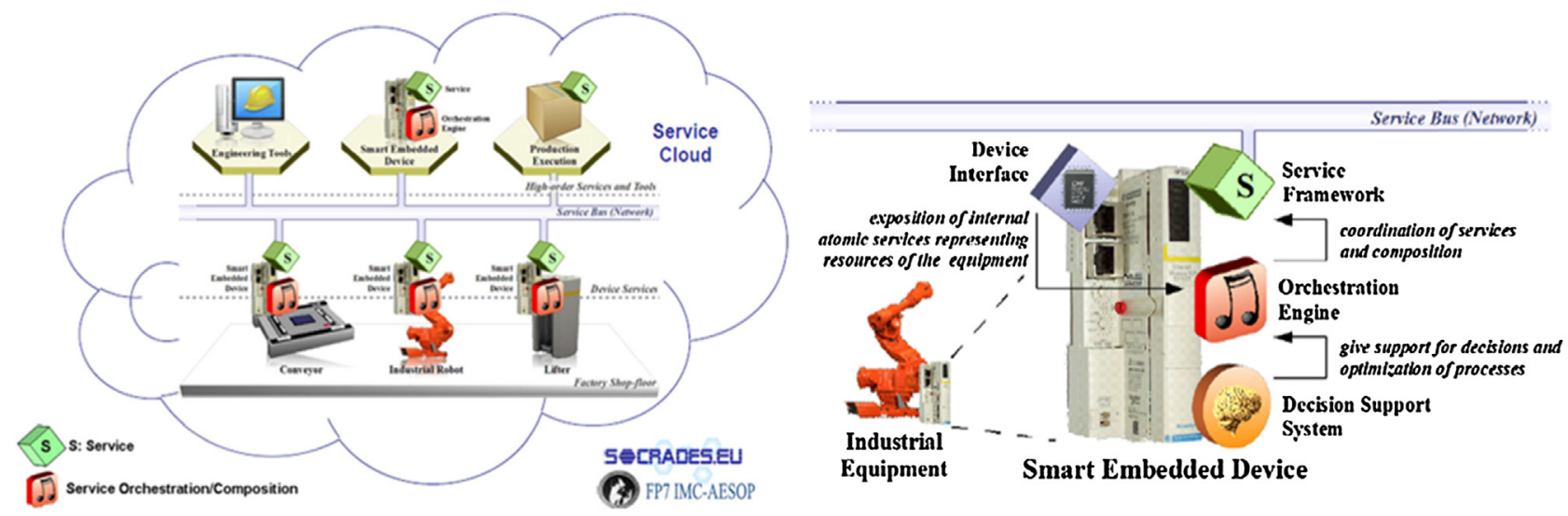

Fig. 7. Network of smart embedded devices.

One very important feature of such service-oriented collaborative automation infrastructure is the capability of building ad-hoc new functionalities in any of the members of the SOA-enterprise, i.e. inside physical but also inside the cyber part of the ICPS. Under the semantic definition of Composition-of-Services, Orchestrationof-Services and/or Choreography-of-Services, it is possible to identify a SOA-based component called "Orchestration Engine" within the automation architecture. This new object is able to generate new services by composing existing/exposed services in the cloud. Represented by a "music-symbol" in Fig. 7, this orchestration engine can play the role of a monitoring component, a control component, a data-analysis-component, etc. There are different formalisms to model and implement the orchestration procedure. In SOCRADES, a kind of Petri nets [48] tailored for formalizing orchestration mechanisms was developed and prototyped, embedding the engine into smart embedded $\mathrm{I} / \mathrm{O}$ devices of the SOA-technology provider Schneider Electric Automation. More information about the Petri net modeling tool and the capabilities of the embedded orchestration engine, is analyzed by Mendes et al. [49], while the integration with enterprise systems is analyzed by Karnouskos et al. [47].

The biggest impact of the SOCRADES project outcomes has been the many lessons learned about how to specify, develop, commission and operate a fully distributed, smart service-oriented collaborative automation architecture, which guarantee the fulfilling of the requirements of flexibility and fast reconfigurability. Although these aspects cannot be expanded in detail here due to space requirements, several examples have been published that demonstrate the benefits of the SOCRADES approach e.g. Mendes et al. [49], Karnouskos et al. [50], Karnouskos et al. [47], Colombo and Karnouskos [9]. In addition, some videos explaining the benefits and hands-on examples are available in YouTube ${ }^{2}$. The gist is that servicification of the infrastructure enables a wide arrange of benefits including reconfiguration, realization of more sophisticated scenarios, cross-layer integration, light-weight implementations etc. all of which have business, technical and operational impacts.

The benefits of service-orientation are conveyed all the way from the upper level of the enterprise architecture, e.g., ERP/MES components to the device level, facilitating the discovery and composition of applications by reconfiguring rather than reprogramming [47]. The dynamic self-configuration of smart embedded devices and systems using loosely-coupled services provides significant advantages for highly dynamic and ad hoc distributed applications, as opposed to the use of more rigid technologies, such

\footnotetext{
${ }^{2}$ EU SOCRADES videos: http://www.youtube.com/watch?v=BCcqb8cumDg, http://www.youtube.com/watch?v=K80tFD6RLMM.
}

as those based only on distributed objects. Moreover, applying the collaborative automation paradigm [8] typically means that all the participating groups in the automation value chain such as control vendors, machine builders and system integrators are confronted with the subject to migrate from legacy hierarchical and completely time-synchron systems to systems composed of building blocks able to run in time-asynchronous mode. The modularization of the automation system requires the decomposition of the present "controller-oriented structure" into functional modules with a "task-oriented structure".

Nowadays, even if SOA as a paradigm and web services as technologies are well-known and even if several applications are available at enterprise level, showing a readiness level 7 and sometimes 8 , the adoption of them at device and system level along the life cycle of automation systems is still at earlydevelopment phases, showing a TRL between 5 and 6 . A deep technology screening and benchmarking allows the following allocation of TRL for the different outcomes of the SOCRADES approach: atomic automation of Web services (TRL 8), composed automation services (TRL 7), automation software as a service (TRL 8/TRL7), orchestration technologies (TRL 6), reconfigurability of SOCRADES-based automation systems (TRL 6) and flexibility of SOCRADES-based automation systems (TRL 6).

\subsection{GRACE}

The GRACE project [10] had the objective to integrate process and quality control using the MAS principles to implement dynamic self-adaptation procedures and feedback control loops, aiming to improve the production efficiency and the product quality. The designed agent-based solution, accommodating selfadaptation and self-optimization mechanisms, was deployed in a real industrial plant producing laundry washing machines [51]. A set of agents were designed for this purpose: Product Type Agents (PTA) representing the catalogue of products that can be produced in the plant, Product Agents (PA) representing the production of product instances being produced along the production line, Resource Agents (RA) representing the resources of the production line, namely processing stations, quality control stations and operators, and Independent Meta Agents (IMA) acting at strategic level to provide global optimization to the production system. The agents collect the shop floor data in a distributed manner, and perform data analysis in real-time to dynamically adjust the production variables, namely processing and inspection operations parameters.

Examples of these procedures are the continuous adaptation of local processing/inspection stations, the selection of functional tests, the parameterization of the on-board controller and the 
generation of quality warnings. Particularizing for the adaptation of the functional tests plan, the product agents are continuously interacting with the resource agents along the production line to acquire feedback related to the quality of the performed (processing and inspection) operations over the washing machines. Very near to the end of the production line, product agents apply an algorithm to adapt the plan of tests to be performed in the washing machine according to their production historical data (note that previously, the plan of functional tests is fixed). The use of this adaptation procedure allows to save inspection time (since some tests are not necessary to be performed) and to execute more efficient inspections tests (since some not usual tests are required or a special attention during one test is required).

In such application, the current low-level control based on PLCs running IEC 61131-3 control programs was preserved to ensure real time responsiveness, as illustrated in Fig. 8. The use of agents integrated with physical devices (namely PLCs and LabView ${ }^{\mathrm{TM}}$ applications) allows to build cyber-physical components that are exposing their functionalities through the use of services. A detailed description of the design, installation and achieved results can be found in Leitão et al. [51].

The designed agent-based solution was implemented using the JADE framework [29] and installed in the factory plant by distributing the plethora of agents by 8 computers disposed along the production line, inter-connected by TCP/IP over an Ethernet network. The installation of the multi-agent system infrastructure for integrating process and quality control can be classified as TRL 6 . The results achieved from the operation of the agent-based solution in the factory plant allowed to summarize a set of benefits, mainly related to an increase of the production efficiency, reflected in the customization of the plan of functional tests and optimization of process parameters, and an improvement of the product quality, reflected in the execution of more efficient inspection tests and customization of the on-board controller of each washing machine.

\subsection{IMC-AESOP}

The IMC-AESOP project [11] has envisioned the next generation of Industrial CPS systems that utilize modern architectures and technologies such as SOA and cloud to increase their operational capabilities. This next generation of Industrial CPS systems constitute of the balance between the physical part (hardware and its integration) and the cyber-part (software and more importantly capability exposure as services). The latter, are split into services on-device, i.e. running within the CPS, and innetwork, i.e. other services running outside the CPS such as the cloud or even in other CPS. This trilateral interaction over welldefined APIs enables the CPS to modularize its capabilities and both offer to 3rd parties as well as utilize services independent of their physical location. Such flexibility acts as an enabler for highly sophisticated and flexible CPS in industrial applications, which empower a new industrial infrastructure that can evolve over time and exhibit emergent behavior.

Fig. 9 depicts this new era of Industrial CPS envisioned by IMCAESOP, which is compliant to the ISÁ95 automation "pyramid" view, but complement it with a flat information-driven modern system and enhance its integrability via modern software engineering practices [52]. This is done by expressing the functionalities found in the different ISÁ95 levels as services that subsequently can be realized on-device (running in the CPS) and in-network, e.g., running on the cloud. As such, one can put together easily the functionalities needed for a specific scenario without having to go through complex stacks and integrate among all layers. With this servification realized, modern software engineering practices can be utilized where service-driven integration can be realized and other benefits for design, development, integration, operation and management aspects of complex and large-scale systems can be obtained. By doing so, new applications required by modern fast-paced industrial environments, can be rapidly realized. Additionally, the move towards a modular and service-based infrastructure enables its easier evolution over time, and therefore it can be adapted to future needs.

In order to offer a concrete example of how this can be realized, the next generation of SCADA/DCS systems was chosen as an Industrial CPS [53]. A set of services has been proposed and a proof of concept was realized, exhibiting several of the benefits expected by such a system. However, the emphasis was not put in creating customized services but instead in more general ones that are part

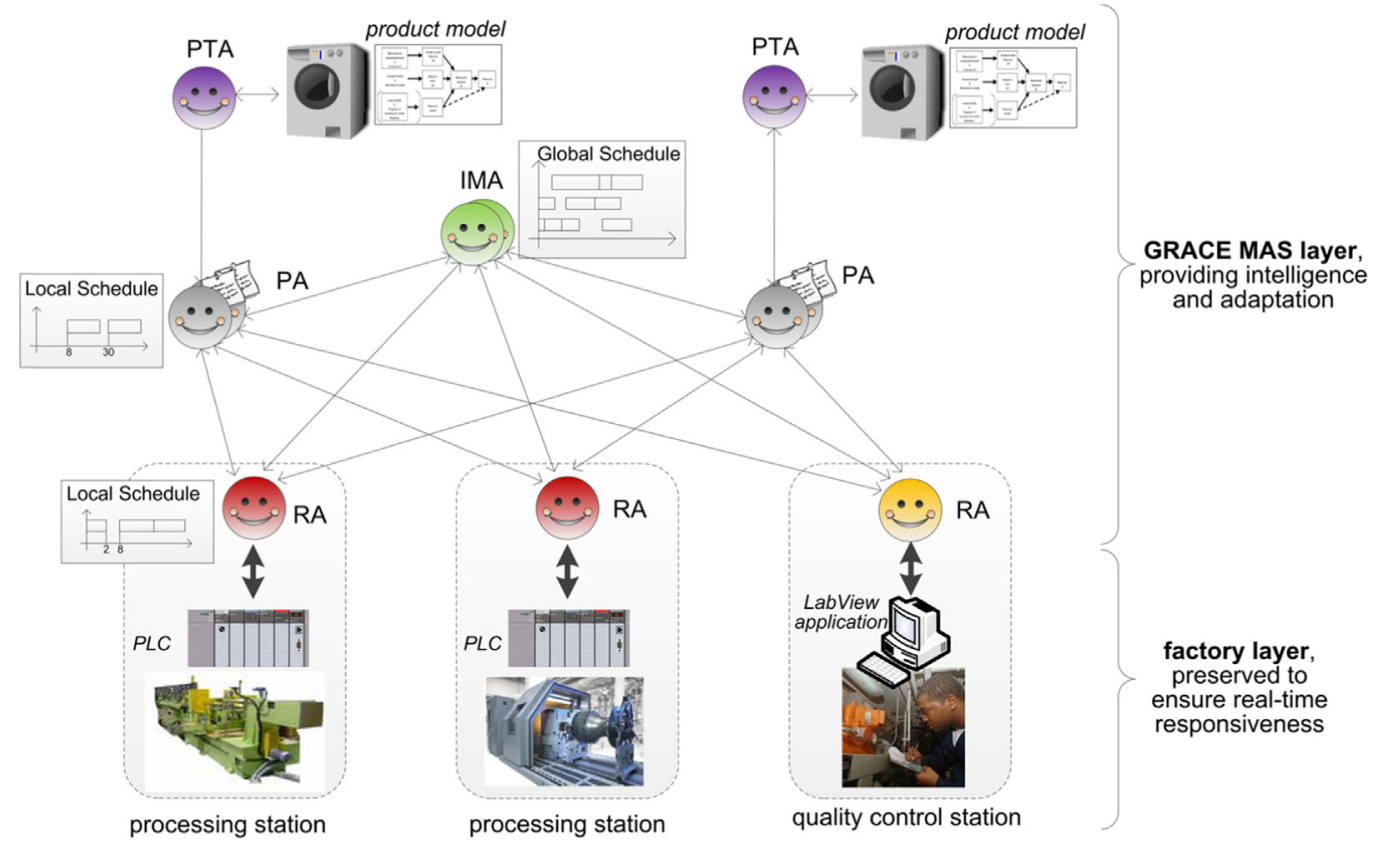

Fig. 8. Multi-agent system for integrating quality and process control. 


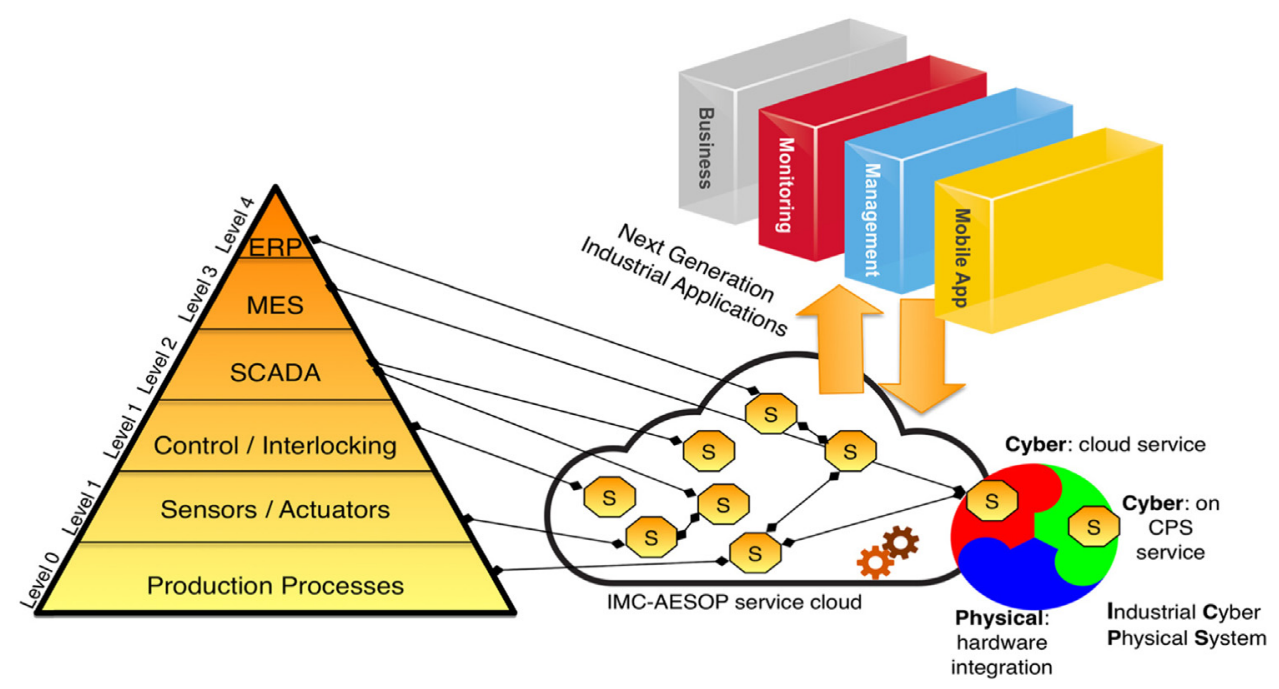

Fig. 9. IMC-AESOP view of cloud-based Industrial CPS.

of the "IMC-AESOP service cloud" and that could be utilized also in other scenarios. Several prototypes demonstrating the feasibility of the approach were realized [11].

IMC-AESOP has proposed a service-oriented architecture [52] that fully utilizes the aforementioned vision of Industrial CPS with functionalities residing on-device and in-cloud. The architecture proposed and realized, is based on the concept of (mostly selfcontained) services, that together build up a common infrastructure, upon which more sophisticated approaches can be built. A detailed description of the architecture, its design considerations, as well as its utilization can be found in Karnouskos et al. [54]. The overall architecture and some services were validated generally in TRL 4, while a subset of these reached TRL 5 and TRL 6. Details on the applications realized based on this architecture, as well as an overview of the relevant technologies, tools and future steps can be found in detail in Colombo et al. [11].

\subsection{ARUM}

The FP7 ARUM project is being developing mitigation strategies to respond faster and more properly to unexpected events in rampup production of complex and highly customized products, such as aircrafts and shipyards, namely in situations of a peak of demand, late changes requests and immature technology for products and processes. For this purpose, agent technology is used to develop production planning, scheduling and optimization tools that expose their internal functionalities as services following the SOA principles. An Enterprise Service Bus (ESB) is used as backbone for supporting the interoperability among the developed knowledge-based MAS decision-support tools. In this case, two ESBs are considered, namely the open source JBoss ESB (www.jboss.org) and the proprietary TIE Smart Bridge (tiekinetix.com). To achieve a full interoperability across the entire solution, these ESBs are enriched with a plethora of advanced modules, such as Ontology service, Data Transformation Service, Sniffer, Node Management and Life-Cycle Management, as illustrated in Fig. 10, resulting on an intelligent ESB. The ESB also allows the integration of legacy systems, such as ERP (Enterprise Resource Planning), MES (Manufacturing Execution System) or SCADA systems.

The plugability of agent-based tools is facilitated by the exposition of their functionalities as services and by the use of ontology services for the representation of the shared knowledge, improving the interoperability in such distributed and heterogeneous systems. As example, strategic planning agent-based tools can compute new planning solutions for a given scene and considering the order demand, operational costs and possible expansion possibilities, e.g., extra shift hours and extra workers, and retrieving legacy data using the Ontology Service from iESB. After the selection of the best solution through a what-if-game process, this solution is passed to the agent-based scheduling tools, through the ESB, to be implemented in the factory plant.

The development of agent-based planning and scheduling tools, interconnected using the service principles, were validated in two use cases. One in the Airbus factory plant of Hamburg addressing the production of the new A350 aircraft and the other one in the Iacobucci factory plant in Ferentino producing modular equipment used during the airplanes' flights, such as coffee machines, trash compactors, ovens and trolleys. Both use cases can be classified as TRL 5. As example, the strategic production planning for the Iacobucci use case was implemented combining the maturity, robustness and optimization of classical mathematical solvers with the flexibility and agility of agent systems. The use of MAS also allows to provide what-if game simulation to support the decisionmakers to take strategic planning decisions on short time with higher confidence. In this use case, the planning agents use the ILOG CPLEX Optimizer solver to run the optimization techniques that solves the planning problem. A detailed description of the design and achieved results of using this strategic planning tool in an integrated manner can be found in Leitão et al. [55].

\section{Discussion}

At this stage, the experience gathered in developing and implementing such kind of CPS systems within the four European research projects allow to perform a critical analysis of the developed use cases and point out scientific and particularly technological challenges, opportunities and road blockers for approaching the increase of the TRLs of CPS solutions.

\subsection{Comparative view of the use cases}

A comparative and critical analysis of the achievements of these research projects is performed in Table 1 focusing different covered aspects: technologies, automation components and functions, and application domains.

The use of MAS, SOA and cloud as key technologies to implement CPS solutions were considered in the four featured European research projects that act as use-cases here. In some situations these technologies were integrated in an isolated manner for a proof of a feature, while in other in a more combined/enabling way that tightly 


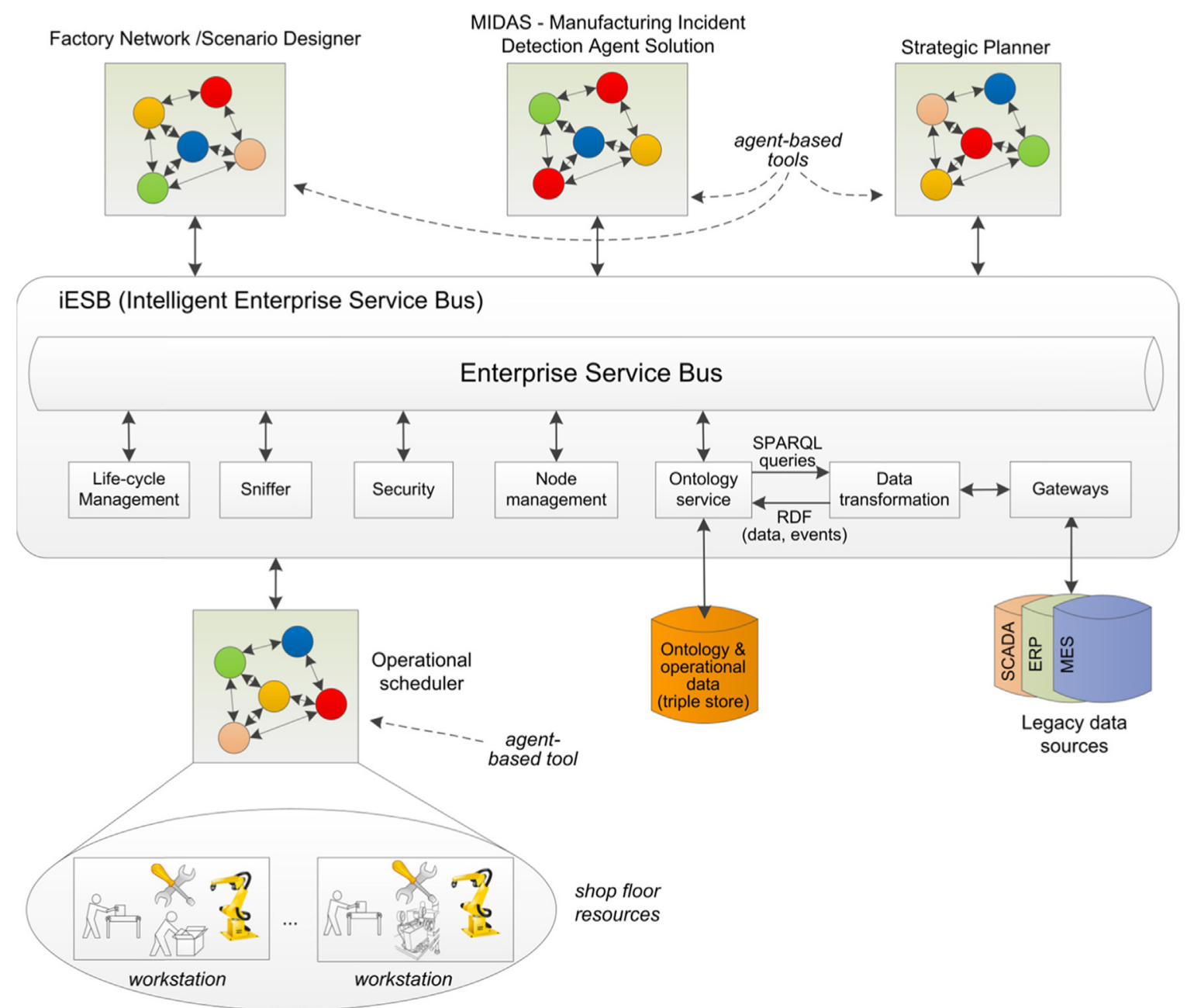

Fig. 10. Agent-based planning and scheduling tools integrated using an ESB infrastructure.

Table 1

Overview of aspects covered by the reference projects.

\begin{tabular}{|c|c|c|c|c|c|}
\hline Key aspects & & $\begin{array}{l}\text { SOCRADES } \\
(2006-2009)\end{array}$ & $\begin{array}{l}\text { GRACE } \\
(2010-2013)\end{array}$ & $\begin{array}{l}\text { IMC-AESOP } \\
(2010-2013)\end{array}$ & $\begin{array}{l}\text { ARUM } \\
(2012-2015)\end{array}$ \\
\hline Technologies & $\begin{array}{l}\text { SOA-based CPS } \\
\text { MAS-based CPS } \\
\text { Cloud-based CPS }\end{array}$ & 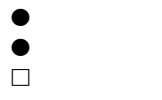 & • & $\begin{array}{l}\bullet \\
\square \\
\bullet\end{array}$ & $\begin{array}{l}\bullet \\
\bullet \\
\square\end{array}$ \\
\hline $\begin{array}{l}\text { Automation components and } \\
\text { functions (according to the } \\
\text { ISA'95 enterprise standard } \\
\text { architecture in Fig. 9) }\end{array}$ & $\begin{array}{l}\text { CPS-based automation control (L1) } \\
\text { CPS-based supervisory control (L2) } \\
\text { CPS-based planning and scheduling (L3) } \\
\text { CPS-based integration in enterprise } \\
\text { systems (L3/L4) }\end{array}$ & $\begin{array}{l}0 \\
\square \\
0 \\
0\end{array}$ & $\begin{array}{l}\bullet \\
\square\end{array}$ & ? & $\stackrel{\bullet}{\bullet}$ \\
\hline Application Domains & $\begin{array}{l}\text { Manufacturing } \\
\text { Electronics assembly } \\
\text { Continuous processing } \\
\text { Energy management }\end{array}$ & $\stackrel{\bullet}{\bullet}$ & $\bullet$ & 0 & $\bullet$ \\
\hline
\end{tabular}

-Covered; $\square$-partially covered.

couples it to the architecture and vision. As the projects presented here span approximately a decade of developments, clearly the technologies and concepts represent also a wider spectrum with respect to design and implementation as well as the maturity and focus.

The usage of SOA in the integration of CPS has been addressed in SOCRADES, IMC-AESOP and ARUM. SOCRADES focused on the integration of devices and CPS in shop-floor as well as with the enterprise systems [47]. IMC-AESOP covered this also by integrating devices, systems, and especially SCADA/DCS systems via on-device and on-cloud services [53]. ARUM addressed the integration of planning and scheduling systems, as well as with legacy enterprise systems and data.

At present besides the benefits that come from the adoption of the SOA approach and associated technologies, some disadvantages/ barriers have been clearly identified. These can be considered challenges that should be addressed and overcome in further developments and exploitation steps. The latter are technology and 
human/user related. Technology-wise, there is not enough readiness to respond to industrial requirements, particularly those requirements associated with the need for hard real-time application. In addition, a common standard for implementing the SOA paradigm along the whole architecture (from shop-floor to enterprise), that will be accepted from ERP and MES developers but also from SCADA and Control suppliers is still not tackled. In an effort to bridge this gap, SOCRADES used DPWS (Device-Profile-for-Web-Services) and made first studies about usage of OPC-UA [56], as key technologies. On the human/user-related side, there is inertia in introducing the approach and technologies in the conservative automation ecosystem characterized by the use of the ISÁ95 enterprise reference architecture. In addition a better understanding of the completely different form of interaction between machines and humans, when both are having similar capabilities, i.e., service exposition or consume, autonomous decision-making and collaborative functions is still not adequately explored. Finally, a well-established migration methodology, approach and adequate engineering tools to transform legacy shop floor into a SOCRADES-compliant infrastructure are still to be developed.

MAS-based CPS has been used partly in IMC-AESOP where agents were used to control a CPS, while they have been of strong focus in SOCRADES, GRACE and ARUM. In these cases, agents have been used for planning, controlling, monitoring and even simulating CPS and large CPS infrastructures.

Cloud-based CPS has been mostly the focus of IMC-AESOP where the next generation of industrial CPS architecture and services have been elaborated [52]. Nevertheless, efforts in other projects such as ARUM and SOCRADES have also partly tackled this area. For instance, although not explicitly named, SOCRADES had developed a SOA-based infrastructure for integration with enterprise services via a specifically developed middleware hosted on an Internet server, what can be considered of an early constrained version of cloud functionalities almost a decade ago.

With respect to automation, SOCRADES and IMC-AESOP focused on integrating devices in L1 according to the ISA'95 automation pyramid (as depicted in Fig. 9). However, this integration was done by wrapping device functionalities and offering the latter as services. Automation devices at L2, such as SCADA/DCS, have been the core focus of IMC-AESOP who envisioned their next generation [53], as well as GRACE for the monitoring and optimization of the feedback control loops at the shop floor level, and partially also SOCRADES with respect to their servification.

CPS-based planning and scheduling (L3) has been the focus on SOCRADES, IMC-AESOP and ARUM. For instance in SOCRADES, the dynamic production planning on disparate factories was realized by integrating real-time events from the production systems. In IMCAESOP, energy management was realized in a greater context by including except from technical and also business objectives and optimizing from the enterprise viewpoint. In ARUM the integration of a new generation of dynamic planning and scheduling tools allow to respond rapidly to unexpected events during the ramp-up production of complex and highly customized products.

Finally, the enterprise integration has been a key aspect targeted mostly in SOCRADES, IMC-AESOP and ARUM, as well as partially in GRACE. The focus on SOCRADES, IMC-AESOP and ARUM was the close integration and interaction of CPS and the industrial processes they manage, with enterprise systems in order to be in tandem with the business goals/objectives and the real-world conditions.

Manufacturing is a well-known area where CPS aspects have been investigated, and this is the case for SOCRADES, GRACE and ARUM. In addition electronics assembly has been demonstrated in SOCRADES over the SOA-based infrastructure. The IMC-AESOP has developed a generic architecture and services for the cloud-based industrial CPS, and demonstrated it in continuous processes and the energy domain. Energy management has been also partially tackled in SOCRADES with focus on measuring and then optimizing energy aspects in production systems and their products [50].

\subsection{Key CPS challenges}

To achieve a wider adoption in industrial environments of the CPS and the technologies that they empower, such as SOA, MAS and Cloud technologies for automation and management of industrial systems, several challenges need to be effectively addressed. Table 2 provides a high level overview of some areas and points to key challenges. We have to point out that Table 2 provides a "rule of thumb" with some preliminary estimations as these are understood based on our experiences, and therefore should be seen with a grain of salt. It is meant to be a discussion proposal to the community and provide a feeling on the key challenges as well as timeline and priorities.

The challenges are roughly clustered in 6 major areas: (i) CPS Capabilities, (ii) CPS Management, (iii) CPS Engineering, (iv) CPS Ecosystems, (v) CPS Infrastructures and (vi) CPS Information Systems. Based on the research and practical experiences of the authors, a ranking of difficulty and a priority level for approaching are defined. Taking into account the latest reported results of scientific and technical developments and evaluating the current state of technologies related to the challenges, a time-horizon to increase the TRLs to at least level 7 (achieve maturity) is forecasted.

On CPS capabilities area, we see that there are several issues related to real-time monitoring and control in CPS as well as CPS SoS (System of Systems) and their optimization which are seen as highly challenging. Their priorities range from medium to high, and especially the control aspects are crucial for the acceptance of CPS in industrial production facilities. There are also other aspects with lower degrees of difficulty, which are partially even possible today e.g. the servification of CPS, however these are also seen as of high priority as they act as enablers for further CPS developments.

On CPS management area, the security and trust coupled with management of (very) large scale CPS is seen as challenging and of high priority. Although industrially mature solutions are expected in the mid to long term, the tackling of the challenges they pose is also expected to be instrumental to the CPS acceptance.

CPS engineering is seen as a priority especially by industry practitioners who will design and deploy future CPS-enabled landscapes. To that extend several highly challenging aspects needs to be addressed, including safe programming and validation, achievement of resilience and graceful degrading, as well as the development of new tools and methods. With the high degree of hardware heterogeneity, new developments in operating systems and programming languages tailored to CPS may arise, which however also may be tackled by significantly extending existing approaches.

In CPS Infrastructures several challenges are identified, however their difficulty level is seen as medium as significant efforts are already underway on how to tackle them. Nevertheless, even if some of them might be more trivial, it is mandatory to resolve them in order to be able to act as enablers for the CPS; a typical example is the interoperable CPS services which have effects on the wider adoption of CPS and their integration in application scenarios.

CPS Ecosystems will increasingly be in the focus as the networking among CPS will prevail. In this area, the key challenges would be to design, deploy, and deal with collaborative, autonomic, self-* and emergent behaviors of CPS. The latter includes all interactions with the environment and the involved stakeholders, including the space of the CPS-human interaction. Although many of these are expected to be maturing only in the 
Table 2

Key challenges in Industrial CPS.

\begin{tabular}{|c|c|c|c|c|}
\hline Area & Key Challenges & Difficulty & Priority & Maturity in \\
\hline \multirow[t]{6}{*}{ CPS Capabilities } & Real-time control of CPS systems & High & High & $4-7$ years \\
\hline & Real-time CPS SoS & High & Medium & $3-5$ years \\
\hline & Optimization in CPS and their application & High & Medium & $4-7$ years \\
\hline & On-CPS advanced analytics & Medium & High & $3-5$ years \\
\hline & Modularization and servification of CPS & low & High & $3-5$ years \\
\hline & Energy efficient CPS & Medium & Medium & $3-5$ years \\
\hline \multirow[t]{3}{*}{ CPS Management } & Lifecycle management of CPS & Medium & Medium & $5-8$ years \\
\hline & Management of (very) large scale CPS and CPS-SoS & High & High & $5-8$ years \\
\hline & Security and trust management for heterogeneous CPS & High & High & $5-8$ years \\
\hline \multirow[t]{5}{*}{ CPS Engineering } & Safe programming and validation of CPS SoS & High & High & $5-10+$ years \\
\hline & Resilient risk-mitigating CPS & High & High & $5-10+$ years \\
\hline & Methods and tools for CPS lifecycle support & High & High & $3-7$ years \\
\hline & New operating systems and programming languages for CPS and CPS SoS & Medium & Low & $3-6$ years \\
\hline & Simulation of CPS and of CPS-SoS & Medium & High & $3-6$ years \\
\hline \multirow[t]{5}{*}{ CPS Infrastructures } & Interoperable CPS services & Medium & High & $2-5$ years \\
\hline & Migration solutions to emerging CPS infrastructures & Medium & High & $3-6$ years \\
\hline & Integration of heterogeneous/mobile hardware and software technologies in CPS & Low & Medium & $2-4$ years \\
\hline & Provision of ubiquitous CPS services & Medium & Medium & $3-5$ years \\
\hline & Economic impact of CPS Infrastructure & High & High & $3-6$ years \\
\hline \multirow[t]{4}{*}{ CPS Ecosystems } & Autonomic and self-* CPS & High & Medium & $7-10+$ years \\
\hline & Emergent behavior of CPS & High & Medium & $7-10+$ years \\
\hline & CPS with humans in the loop & High & High & $2-5$ years \\
\hline & Collaborative CPS & Medium & Medium & $5-8$ years \\
\hline \multirow[t]{4}{*}{ CPS Information Systems } & Artificial intelligence in CPS & High & High & $7-10+$ years \\
\hline & Cross-domain large-scale information integration to CPS infrastructures & Medium & Low & $6-9$ years \\
\hline & Transformation of CPS data and information analytics to actionable knowledge & High & High & $4-8$ years \\
\hline & Knowledge-driven decision making/management & High & Medium & $6-10+$ years \\
\hline
\end{tabular}

long-run, the path of dealing with these challenges is not straightforward.

CPS Information Systems will be able to capitalize on the data, information and knowledge acquired, however to do so several key challenges need to be resolved in order to make that possible. As such actions are mostly in the long term, and their difficult is not trivial, the priorities vary. At this stage the industry is mostly interested in the mid-term in transforming CPS data and information analytics to actionable knowledge, while the crossdomain information integration and knowledge-driven decision making/management are seen as follow-up actions.

\section{Conclusions}

CPS provides the necessary technological basis to facilitate the realization and corresponding automation of large-scale complex system, such as smart grids, smart buildings, smart transportation, smart healthcare and smart manufacturing, among other application areas. The CPS era is in need of solutions that will support it at device, system, infrastructure and application level. This includes the whole lifecycle from cradle-to-grave of its CPS components and services. This is a scientific, technical, industrial and social challenge that includes a multi-disciplinary engineering approach and the confluence and sometimes fusion of heterogeneous communication, information and control/automation technologies (see Ref. [57]). This work has presented an overview of key aspects related to Industrial CPS and key approaches and technologies associated with their engineering and implementation related to industrial automation, such as MAS, SOA and cloud systems. Based on the results of four European innovation projects (i.e. SOCRADES, IMC-AESOP, GRACE and ARUM), the progress in the domain has been reported. Subsequently, key challenges for the understanding and application of industrial automation based on CPS technologies have been identified and some considerations on the difficulties and time horizon are discussed, with the aim to support further the increasing of the current Technology Readiness
Levels and lead to a broad utilization of CPS-based systems and infrastructures in commercial industrial automation systems.

\section{Acknowledgements}

The authors would like to thank for their support the European Commission, and the partners of the EU FP6 SOCRADES (www. socrades.net), EU FP7 GRACE (www.grace-project.org), EU FP7 IMCAESOP (www.imc-aesop.eu) and EU FP7 ARUM (www.arum-project. eu) projects, for their fruitful support and discussions.

\section{References}

[1] H. ElMaraghy, Flexible and reconfigurable manufacturing systems paradigms, Int. J. Flexible Manuf. Syst. 17 (2006) 261-271.

[2] P.C. Evans, M. Annunziata, Industrial Internet: Pushing the Boundaries of Minds and Machines, Whitepaper, General Electric, 2012 /http://www.ge.com/ docs/chapters/Industrial_Internet.pdf).

[3] H. Kagermann, W. Wahlster, J. Helbig, Securing the future of German manufacturing industry: recommendations for implementing the strategic initiative INDUSTRIE 4.0, in: Technical Report, German National Academy of Science and Engineering (ACATECH), 2013 /http://www.acatech.de/fileadmin/ user_upload/Baumstruktur_nach_Website/Acatech/root/de/ Material_fuer_Sonderseiten/Industrie_4.0/ Final_report_Industrie_4.0_accessible.pdf $\rangle$

[4] BMBF, The New High-Tech Strategy Innovations for Germany, 2014 Available at 〈http://www.bmbf.de/pub/HTS_Broschuere_engl_bf.pdf $\rangle$.

[5] BMBF, Autonomics for Industry 4.0, 2015 Available at 〈http:// www.autonomik40.de/en/>.

[6] NIST, Cyber-Physical Systems: Situation Analysis of Current Trends, Technologies, and Challenges, National Institute of Standards and Technology (NIST), Columbia, Maryland, 2012, 〈http://events.energetics.com/NISTCPSWorkshop/pdfs/CPS_Situation_Analysis.pdf $/$.

[7] H. Sundmaeker, P. Guillemin, P. Fries, S. Woelffle, Vision and challenges for realising the internet of things, in: CERP-IoT Cluster of European Research Projects on the Internet of Things, 2010.

[8] A.W. Colombo, S. Karnouskos, T. Bangemann, A system of systems view on collaborative industrial automation, in: IEEE International Conference on Industrial Technology (ICIT 2013), 25-28 Feb, Cape Town, South Africa, 2013.

[9] A.W. Colombo, S. Karnouskos, Towards the factory of the future: a serviceoriented cross-layer infrastructure, in: ICT Shaping the World: a Scientific View, European Telecommunications Standards Institute (ETSI), Wiley, New York, NY, 2009, pp. 65-81 
[10] C. Cristalli, M. Foehr, T. Jäger, P. Leitao, N. Paone, P. Castellini, C. Turrin, I. Schjolberg, Integration of process and quality control using multi-agent technology, in: Proceedings of the 22nd IEEE Int. Symposium on Industrial Electronics. (ISIE'13), 2013.

[11] A.W. Colombo, T. Bangemann, S. Karnouskos, J. Delsing, P. Stluka, R. Harrison, F. Jammes, J.M. Lastra, Industrial Cloud-based Cyber-Physical Systems: The IMC-AESOP Approach, Springer International Publishing, Switzerland, 2014, , ISBN: 978-3-319-05623-4.

[12] C. Marín, L. Mönch, P. Leitão, P. Vrba, D. Kazanskaia, V. Chepegin, L. Liu, N. Mehandjiev, A conceptual architecture based on intelligent services for manufacturing support systems, in: Proc. of the IEEE International Conference. on Systems, Man, and Cybernetics (SMC'13), 2013, 4749-4754.

[13] E.A. Lee, S.A. Seshia, Introduction to Embedded Systems-A Cyber-Physical Systems Approach, Lulu.com, 2013 13: 978-0557708574 〈http://leeseshia.org/〉.

[14] P.J. Marrón, D. Minder, S. Karnouskos, The Emerging Domain of Cooperating Objects: Definition and Concepts, Springer-Verlag Berlin, Heidelberg, 2012, , ISBN: 978-3-642-28469-4.

[15] A.V. Vasilakos, P. Manish, S. Karnouskos, W. Pedrycz, Autonomic Communication, Springer Science + Business Media, 2010, , http://dx.doi.org 10.1007/978-0-387-09753-4, ISBN: 978-0-387-09752-7.

[16] R. Schoop, R. Neubert, A.W. Colombo, A multiagent-based distributed control platform for industrial flexible production systems, in: Proc. of the 27th Annual Conference of IEEE Industrial Electronics (IECOŃ01), 2001, 279-284.

[17] M. Wooldridge, An Introduction to Multi-Agent Systems, JohnWiley \& Sons, West Sussex, England, 2002.

[18] P. Leitão, Agent-based distributed manufacturing control: a state-of-the-art survey, Eng. Appl. Artif. Intell. 22 (7) (2009) 979-991 (2009).

[19] P. Leitão, S. Karnouskos, Industrial Agents: Emerging Applications of Software Agents in Industry, Elsevier, 2015 13: 978-0128003411.

[20] T. Gruber, Toward principles for the design of ontologies used for knowledge sharing, Int. J. Hum. Comput. Stud. 43 (5/6) (1995) 907-928.

[21] A. Koestler, The Ghost in the Machine, Arkana Books, London, 1969.

[22] A.W. Colombo, R. Neubert, R. Schoop, A solution to holonic control systems, in: Proc. of the eighth IEEE Int. Conf. on Emerging technologies and Factory Automation (ETFA'01), 2001, 489-498

[23] L. Ribeiro, J. Barata, A.W. Colombo, MAS and SOA: a case study exploring principles and technologies to support self-properties in assembly systems, in: Proc. of the Second IEEE Workshop on Self-Adaptive and Self-Organizing Systems (SASOW'08), 2008, 192-197.

[24] P. Leitão, A.W. Colombo, F. Restivo, An Approach to the Formal Specification of Holonic Control Systems, Holonic and Multi-Agent Systems for Manufacturing, Lecture Notes in Artificial Intelligence, Springer, Berlin Heidelberg, 2003, pp. 59-70.

[25] V. Marik, D. McFarlane, Industrial adoption of agent-based technologies, IEEE Intell. Syst. 20 (1) (2005) 27-35.

[26] M. Winkler, M. Mey, Holonic manufacturing systems, Eur. Prod. Eng. 3-4 (1994) $10-12$.

[27] A.W. Colombo, S. Karnouskos, J.M. Mendes, P. Leitão, Industrial agents in the era of service oriented architectures and cloud based industrial infrastructures, in: Industrial Agents: Emerging Applications of Software Agents in Industry, Elsevier, 2015 13: 978-0128003411.

[28] G. Candido, A.W. Colombo, J. Barata, F. Jammes, Service-oriented infrastructure to support the deployment of evolvable production systems, IEEE Trans. Ind. Inf. 7 (4) (2011) 759-767.

[29] F. Bellifemine, G. Caire, D. Greenwood, Developing Multi-Agent Systems with JADE, Wiley, West Sussex, England, 2007.

[30] J.M. Mendes, P. Leitão, F. Restivo, A.W. Colombo, Service-oriented agents for collaborative industrial automation and production systems, in: V. Marik, T Strasser, A. Zoitl (Eds.), Proceedings of the Fourth International Conference on Industrial Applications of Holonic and Multi-Agent Systems (HoloMAS'09), Lecture Notes in Artificial Intelligence, vol. 5696, Springer-Verlag Berlin, Heidelberg, 2009, pp. 13-24.

[31] P. Leitão, S. Karnouskos, A survey on factors that impact industrial agents acceptance, in: Industrial Agents: Emerging Applications of Software Agents in Industry, Elsevier, 2015 13: 978-0128003411.

[32] L. Monostori, J. Váncza, S. Kumara, Agent-based systems for manufacturing Ann. CIRP 55/2 (2006) 697-720.

[33] M. Pechoucek, V. Marik, Industrial deployment of multi-agent technologies: review and selected case studies, Auton. Agents Multi-agent Syst. 17 (13) (2008) 397-431.

[34] L. Badger, T. Grance, R. Patt-Corner, J. Voas, Cloud Computing Synopsis and Recommendations, National Institute of Standards and Technology (NIST), 2012 〈http://csrc.nist.gov/publications/nistpubs/800-146/sp800-146.pdf〉 (Special publication 800-146)

[35] M. Chen, S. Mao, Y. Liu, Big data: a survey, Mobile Netw. Appl. 19 (2) (2014) 171-209, http://dx.doi.org/10.1007/s11036-013-0489-0.

[36] D. McFarlane, V. Giannikas, A.C.Y. Wong, M. Harrison, Product intelligence in industrial control: theory and practice, Annu. Rev. Control 37 (1) (2013) 6988.

[37] Cisco, Fog Computing, Ecosystem, Architecture and Applications, 2013 Available at 〈http://www.cisco.com/web/about/ac50/ac207/crc_new/ university/RFP/rfp13078.html $>$ (accessed on 8th March 2015).

[38] G.A. Boy, Orchestrating Human-Centered Design, Springer-Verlag, London, 2012

[39] P. Miller, The Genius of Swarms, National Geographic, July, 2007.

[40] E. Bonabeau, M. Dorigo, G. Theraulaz, Swarm Intelligence: from Natural to Artificial Systems, Oxford University Press, New York, NY, 1999.
[41] J. Holland, Emergence: From Chaos to Order, Oxford University Press, New York, NY, 1998.

[42] S. Kauffman, The Origins of Order: Self Organization and Selection in Evolution, Oxford University Press, New York, NY, 1993.

[43] S. Bussmann, K. Schild, Self-organizing manufacturing control: an industrial application of agent technology, in: Proceedings of the Fourth International Conference on MultiAgent Systems (ICMAS-2000), Washington, DC, (2000), pp. 87-94.

[44] J. Barbosa, P. Leitão, E. Adam, D. Trentesaux, Dynamic self-organization in holonic multi-agent manufacturing systems: the ADACOR evolution, Comput. Ind. 66 (2015) 99-111.

[45] European Commission, Technology readiness levels (TRL), European Commission, HORIZON 2020 work programme 2014-2015 general annexes, in: Extract from Part 19-Commission Decision C(2014)4995, 2014 (http:// ec.europa.eu/research/participants/data/ref/h2020/wp/2014_2015/annexes/ h2020-wp1415-annex-g-trl en.pdf).

[46] R. Harrison, A.W. Colombo, Collaborative automation from rigid coupling towards dynamic reconfigurable production systems, IFAC World Congr. 16 (1) (2005).

[47] D. Savio Karnouskos, P. Spiess, D. Guinard, V. Trifa, O. Baecker, Real world service interaction with enterprise systems in dynamic manufacturing environments, in: L. Benyoucef, B. Grabot (Eds.), Artificial Intelligence Techniques for Networked Manufacturing Enterprises Management, Springer, London, 2010, , ISBN: 978-1-84996-118-9.

[48] T. Murata, Petri nets: properties, analysis and applications, IEEE 77 (4) (1989) $541-580$.

[49] J.M. Mendes, A. Bepperling, J. Pinto, P. Leitão, F. Restivo, A.W. Colombo, Software methodologies for the engineering of service-oriented industrial automation: the continuum project, in: Proc. of the 33rd Computer Software and Applications Conference (COMPSAC'09), 2009, 452-459.

[50] S. Karnouskos, A.W. Colombo, J.L. Martínez Lastra, C. Popescu, Towards the energy efficient future factory, in: Seventh IEEE International Conference on Industrial Informatics INDIN 2009, 23-26 Jun 2009, Cardiff, UK, (2009), pp. 367-371, http://dx.doi.org/10.1109/INDI.N.2009.5195832.

[51] P. Leitão, N. Rodrigues, C. Turrin, A. Pagani, Multi-agent system for integrating quality and process control in a home appliance production line, in: P. Leitão, S. Karnouskos (Eds.), Industrial Agents: Emerging Applications of Software Agents in Industry, Elsevier, Amsterdam, 2015.

[52] S. Karnouskos, A.W. Colombo, T. Bangemann, K. Manninen, R. Camp, M. Tilly, P. Stluka, F. Jammes, J. Delsing, J. Eliasson, A SOA-based architecture for empowering future collaborative cloud-based industrial automation, in: 38th Annual Conference of the IEEE Industrial Electronics Society (IECON 2012), 25-28 Oct., Montréal, Canada, (2012), pp. 5766-5772.

[53] S. Karnouskos, A.W. Colombo, Architecting the next generation of service-based SCADA/DCS system of systems, in: 37th Annual Conference of the IEEE Industria Electronics Society (IECON 2011), 7-10 November, Melbourne, Australia, 2011.

[54] S. Karnouskos, A.W. Colombo, T. Bangemann, K. Manninen, R. Camp, M. Tilly, M. Sikora, F. Jammes, J. Delsing, J. Eliasson, P. Nappey, J. Hu, M. Graf, The IMCAESOP Architecture for Cloud-based Industrial CPS in Industrial Cloud-Based Cyber-Physical Systems, Springer, 2014, pp. 49-88 Available at: http:// dx.doi.org/10.1007/978-3-319-05624-1_3.

[55] P. Leitão, Nelson Rodrigues, J. José Barbosa, What-if game simulation in agentbased strategic production planners, in: Proceedings of the 20th IEEE International Conference on Emerging Technologies and Factory Automation (ETFA'15), September, 2015

[56] W. Mahnke, S.-H. Leitner, M. Damm, OPC Unified Architecture, Springer Verlag, 2009, http://dx.doi.org/10.1007/978-3-540-68899-0.

[57] R. Schiller, Die Stunde 4.0, Wie das Internet der Dinge unser Leben verändert, Südeutsche Zeitung, Num. 78 (2015) 25-26.

[58] G. Gorbach, R. Mick, Collaborative manufacturing management strategies, in: ARC Strategies, White Paper, November, 2002.

[59] P. Leitão, F. Restivo, ADACOR: a holonic architecture for agile and adaptive manufacturing control, Comput. Ind. 57 (2) (2006) 121-130.

[60] P. Valckenaers Hadeli, M. Kollingbaum, H. Brussel, O. Bochmann, Stigmergy in holonic manufacturing systems, J. Integr. Comput. Aided Eng. 9 (3) (2002) 281-289.

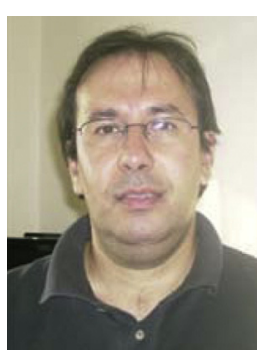

Paulo Leitão received the MSc and $\mathrm{PhD}$ degrees in Electrical and Computer Engineering, both from the University of Porto, Portugal, in 1997 and 2004, respectively. He is Professor at the Polytechnic Institute of Braganç, currently Head of the Department of Electrical Engineering, and member of the Artificial Intelligence and Computer Science Laboratory (LIACC). His research interests are in the field of industria informatics, collaborative factory automation, reconfigurable production systems, cyber-physical systems, intelligent supervisory control, agent-based systems and holonic control. He participate/has participated in several national and international research projects and Networks of Excellence, has published more than 140 papers in high-ranked international scientific journals and conference proceedings (per-review), is coauthor of three patents and served as general co-chair of several international conferences, namely IFAC IMS'10 and HoloMAS'11. Dr. Leitão is Senior member of the IEEE Industrial Electronics Society and currently the Chair of the IEEE Industrial Electronics Society Technical Committee on Industrial Agents 


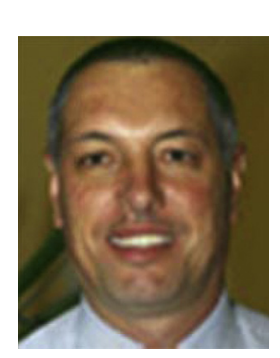

Armando Walter Colombo joined the Department of Electrotechnic and Industrial Informatics at the University of Applied Sciences Emden-Leer, Germany, and became Full Professor in August 2010. He is also Edison Level 2 Group Senior Expert and Program Manager at Schneider Electric. He received the MSc. on Control System Engineering from the National University of San Juan, Argentina, in 1994, and the Doctor degree in Engineering from the University of Erlangen-Nuremberg, Germany, in 1998. From 1999 to 2000 was Adjunct Professor in the Group of Robotic Systems and CIM, Faculty of Technical Sciences, New University of Lisbon, Portugal. Since 2001 Prof. Colombo has been working as Research/Collaborative Program Manager at Schneider Electric. He has extensive experience in managing multi-cultural research teams in multi-regional projects. Prof. Colombo has participated in leading positions in many international projects, e.g. he was co-leader of the RTD-Cluster on Production Automation and Control (PAC) of the EU FP6 NoE IPROMS" (www.iproms.org, 20042009), technical manager of the EU FP6 STREP RI-MACS (2005-2008), the Co-ordinator of the EU FP6 Integrated Project “SOCRADES" (www.socrades.eu, 2006-2009), with the participation of all major European Stakeholders of the Automation value chain, i.e., Schneider Electric, Siemens, ABB, but also ARM, SAP, Jaguar/Ford, etc., and is now coordinating the EU FP7 IP IMC-AESOP (with the participation of 17 European partners: Honeywell, Microsoft, SAP, Schneider Electric, Politecnico di Milano, Tampere Univ. of Technology, Loughborough University, etc., see www.imc-aesop.eu, 2010-1013). Prof. Colombo works also as Invited Lecturer at the Tampere University of Technology, Finland, and is co-supervisor of PhD-Students at the Loughborough University (UK), Porto University (Portugal), Politecnico di Milano (Italy) and New University of Lisbon (Portugal). His research interests are in the fields of cyber-physical systems, system-ofsystems engineering (SoSE), service-oriented architecture (SoA), collaborative automation, intelligent supervisory control, formal specification of flexible automation systems, Petri nets. Prof. Colombo has more than 180 publications (per-review) in journals, books, and chapters of books and conference proceedings. He has 21 industrial patent applications. He is a Senior Member of the IEEE and member of the Gesellschaft für Informatik e.V. Prof. Colombo served/serves as Associated Editor of the IEEE Transactions on Industrial Informatics, IEEE Transactions on Automation Systems Engineering (IEEE T-ASE) and Associated Editor of the IFAC Associated Journal ATPInternational. He is member of the IEEE IES Administrative Committee (AdCom), CoChairman of the IEEE IES Committee on Industrial Informatics. He also served as General Co-Chair in the last issues of the IEEE Conferences on Industrial Informatics (INDIN 2009-2011). Prof. Colombo is the co-leader of the ARTEMIS (European Embedded Systems Platform) Strategic Research Agenda - Sub-Program ASP4. Since 2003 Prof. Colombo served/s as advisor/expert for the definition of the R\&D priorities within the Framework Programs FP6, FP7 and FP8 (HORIZON 2020) of the Research Executive Agency (REA) in the European Union. Prof. Colombo is listed in Who's Who in the World/Engineering 1999-2000/2001 and in outstanding People of the XX Century (Bibliographic Centre Cambridge, UK).

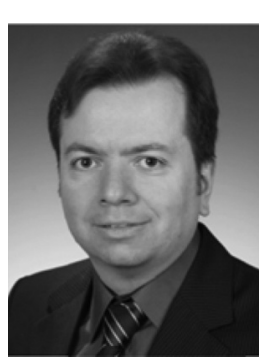

Stamatis Karnouskos is with SAP as a Research Expert on Internet of Things/Cyber-Physical Systems, investigating the added value of their integration with enterprise systems. For more than 18 years Stamatis leads efforts in several European Commission and industry funded projects related to industrial informatics, industrial automation, smart grids, smart cities, Internet-based services and architectures, software agents, mobile commerce, security and mobility. 